\title{
Probing Presynaptic Regulation of Extracellular Dopamine with Iontophoresis
}

\author{
Natalie R. Herr, ${ }^{\dagger}$ Anna M. Belle, ${ }^{\dagger}$ Kevin B. Daniel,${ }^{\dagger}$ Regina M. Carelli, ${ }^{\dagger} \S^{8}$ and \\ R. Mark Wightman*, $*, \$$ \\ ${ }^{\dagger}$ Department of Chemistry, ${ }^{*}$ Department of Psychology, and ${ }^{\S}$ Neuroscience Center, University of North Carolina at Chapel Hill, \\ Chapel Hill, North Carolina 27599-3290
}

\section{Abstract}

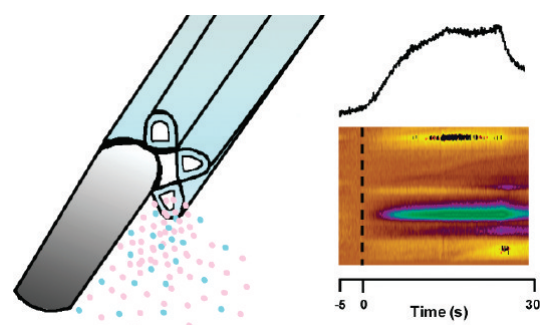

Iontophoresis allows for localized drug ejections directly into brain regions of interest driven by the application of current. Our lab has previously adapted a method to quantitatively monitor iontophoretic ejections. Here those principles have been applied in vivo to modulate electrically evoked release of dopamine in anesthetized rats. A neutral, electroactive marker molecule that is ejected purely by electroosmotic flow (EOF) was used to monitor indirectly the ejection of electroinactive dopaminergic drugs (raclopride, quinpirole, and nomifensine). Electrode placements were marked with an iontophoretically ejected dye, pontamine sky blue. We show that EOF marker molecules, acetaminophen (AP) and 2-(4-nitrophenoxy) ethanol (NPE), have no effect on electrically evoked dopamine release in the striatum or the sensitivity of electrode. Additionally, we establish that a short, $30 \mathrm{~s}$ ejection of raclopride, quinpirole, or nomifensine with iontophoresis is sufficient to affect autoreceptor regulation and the reuptake of dopamine. These effects vary in lifetime, indicating that this technique can be used to study receptor kinetics.

Keywords: Iontophoresis, fast-scan cyclic voltammetry, dopamine, carbon fiber, quantitative, presynaptic

I ontophoresis was first developed by W. L. Nastuk, a student of A. L. Hodgkin, who was interested in how the actions of acetylcholine (ACh) on the neuromuscular junction were altered with changes in the ionic composition of the extracellular bath solution (1). His previous studies with intracellular pipet recordings led him to the discovery that if pipets were pulled to a coarse tip and then filled with ACh, some
ACh would slowly diffuse out. He expanded on this observation and decided to electrically control delivery of ACh through the glass pipettes, and thus iontophoresis was born in 1953 (2). Recognizing that fast and controlled delivery of ACh could be used to search and map end-plate regions on the neuromuscular region, many researchers, including del Castillo and Katz, used it to study the actions of ACh on synaptic sites (3-6). The first studies using iontophoresis in the central nervous system were made by Eccles and Curtis who were interested in studying Renshaw cells and used the first account of a multibarrel iontophoresis probe to locate and modulate cells (7-9).

Throughout the remainder of the 1950s and into the 1970s, iontophoresis grew in popularity, and important contributions concerning the advantages and disadvantages of iontophoretic drug delivery were made (10-19). The technique was (and is) favored for studying receptor dynamics in vivo because drugs can be quickly, selectively, and locally delivered to the site (or sites) of action. Traditional methods of pharmacological intervention, such as intraperitoneal or intravenous delivery, affect the entire brain and can confound interpretation of the measured results. Furthermore, only drugs that can pass the blood-brain barrier can be used for systemic drug delivery, and even then, metabolism of the drug may reduce its effects (15). Iontophoresis circumvents all of these problems, making it very attractive for pharmacological neurobiology studies.

However, despite the clear advantages of iontophoresis, challenges with reproducibility and quantitation of drug delivery have prevented the technique from being more widely used $(3,11,13,15,18-23)$. A major drawback of iontophoresis as it has been previously used is that there is no way to differentiate between an unresponsive site and a faulty drug ejection. Recently, we modified the design of Millar and co-workers, coupling iontophoresis barrels to carbon-fiber microelectrodes to allow the concentrations of electroactive compounds delivered by iontophoresis to be monitored with fast-scan

Received Date: June 2, 2010

Accepted Date: July 13, 2010

Published on Web Date: July 28, 2010 
cyclic voltammetry (22). While characterizing iontophoretic delivery, we found that electroosmosis contributes significantly to the observed drug delivery. Electroosmosis is due to ionizable silanol groups on the glass capillary surface, which attract cations in solution to form an electrical double layer. When a positive current is applied to the capillary, the cations along the wall migrate toward the anode (outside of the capillary), creating a bulk movement of solution, termed electroosmotic flow (EOF). Thus, iontophoretic delivery is governed by the traditional mechanism attributed to iontophoresis, ion migration, and EOF. In addition, we showed that an electroactive neutral molecule could serve as an internal standard to monitor the variability in the amount of drug delivered from different barrels. These insights into the iontophoresis technique enable quantitative delivery of electroactive and electroinactive drugs by monitoring the ejection of an electroactive EOF marker (24).

Although fast-scan cyclic voltammetry has been previously combined with iontophoresis for neurophysiology experiments, it has not been used to modulate presynaptic release of neurotransmitters $(25-31)$. In this paper, we demonstrate the use of quantitative iontophoresis for the modulation of dopamine release in the striatum of anesthetized rats. We characterize ejections in vivo with particular emphasis on leakage and the time course of drug ejection. We also have tested the effects on dopamine release upon application of two electroactive marker molecules, AP and NPE, to ensure that our method of quantitation does not alter the biological system. Expanding on our previous work, we have quantified the relative mobilities of drugs of pharmacological interest that are not electroactive. Finally, we demonstrate that dopamine neurotransmission can be locally modulated at terminals by affecting D2 autoreceptors and the dopamine transporter.

\section{Results and Discussion}

\section{Characterization of Iontophoretic Delivery in Vivo}

Traditionally, iontophoretic ejection of substances with constant current has been considered as continuous electrical migration of an ion out of the pipet tip followed by diffusion into the surrounding environment (32). Theoretical and experimental calculations predict that migration out of the pipet tip will be influenced by the prior history of the pipet including the magnitude of the retaining current applied and the frequency of previous ejections $(18,19)$. Our previous work showed that the iontophoretic barrels that we construct have sufficiently small leakage that it is immeasurable by the adjacent carbon-fiber microelectrode (24). However, we observe that the first few
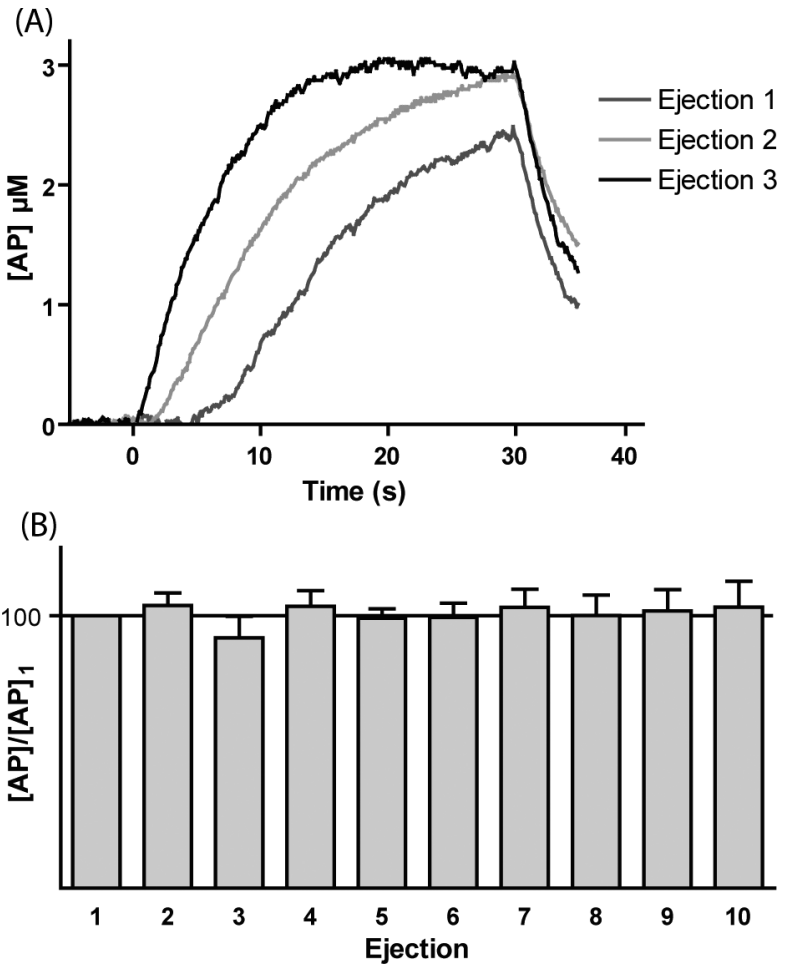

Figure 1. Temporal profile of consecutive iontophoretic ejections (A) Current vs time trace for the first three ejections of AP in the rat brain. Ejection current was turned on at $t=0 \mathrm{~s}$ and off at $t=30 \mathrm{~s}$. Ejection 1 shows a characteristic delay in rise time, consistent with a "warm-up" period for ejections. Subsequent ejections, 2 and 3, have less of a delay, and ejection 3 reaches steady state. (B) 10 consecutive ejections into PBS buffer after "warm-up" period. $[\mathrm{AP}] /[\mathrm{AP}]_{1}$ represents the amount of AP measured at steady state compared with the amount measured from the first ejection after the "warm-up" period.

ejections have diminished ejection efficiency, presumably due to leakage during the time for implantation and stabilization of the electrode $(\sim 1 \mathrm{~h})$. This is shown in Figure 1, where the local AP concentration was monitored at the peak current of its oxidation during fastscan cyclic voltammetry as it was ejected into the striatum of an anesthetized rat. The time course during $30 \mathrm{~s}$ ejections is shown in Figure 1A. The voltammetric response increases when the iontophoretic current is initiated. The response continues to increase as the ejection continues and reaches a steady state. As shown, the time to reach a steady state is longer for the first ejection than the subsequent ones. This delay is the likely cause of the "warm-up phenomenon" noted in previous studies where initial iontophoretic ejections elicited little to no biological response, but with subsequent ejections the response grew in over time (17). For this reason, before beginning biological studies, we "warm up" the electrode by continuously ejecting for $2-5 \mathrm{~min}$ into an area of the brain that is not of interest. Figure 1B shows the reproducibility of ejections after the "warm-up" period. Ten consecutive ejections for a single barrel show a similar steady-state level compared with the first ejection after the warm-up period $(n=5)$. However, to 
(A)

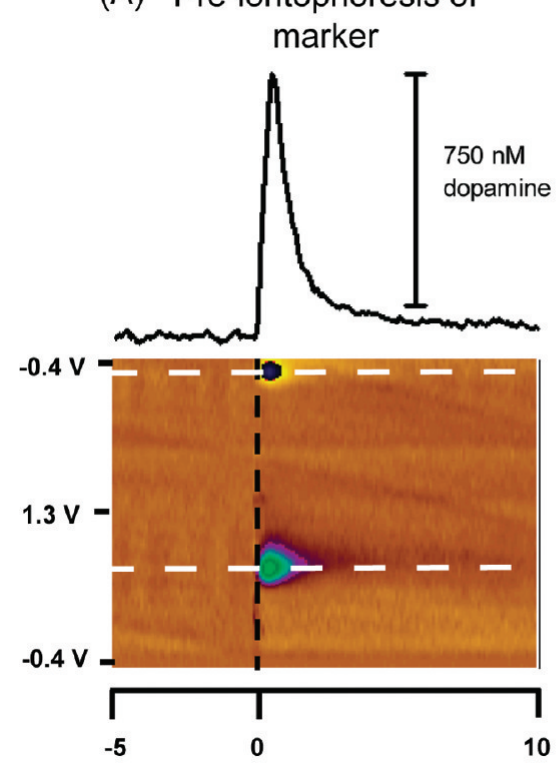

(B)

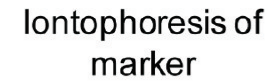

marker

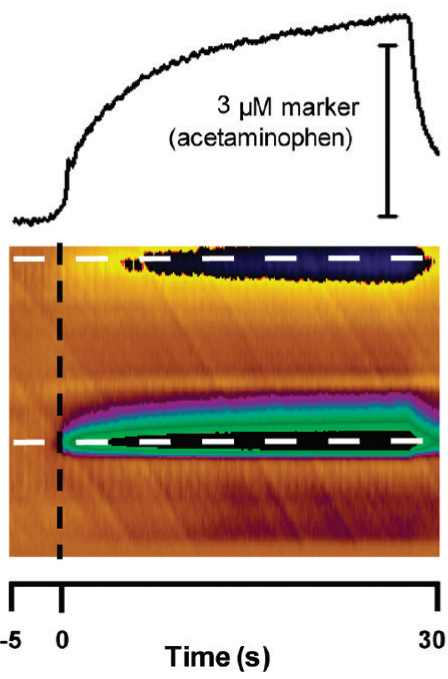

(C) Post-iontophoresis of marker

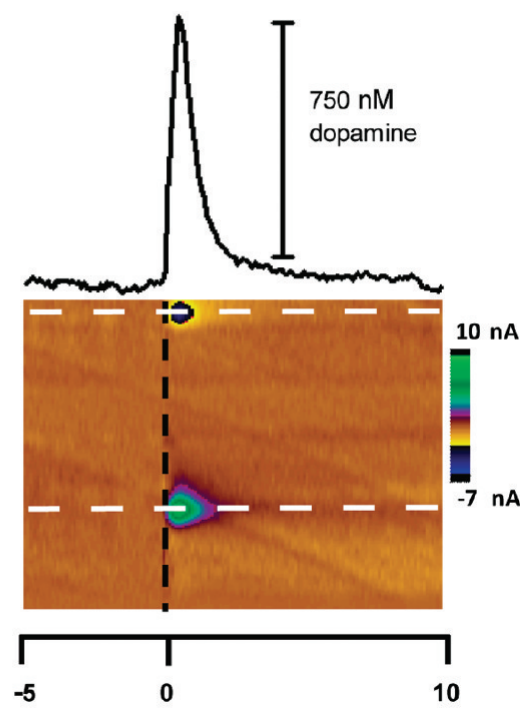

Figure 2. Effect of AP on stimulated dopamine release. The top panels show current as a function of time while the lower panels are twodimensional color plots where current is shown in false color on the potential vs time axes. The white dashed lines on the color plots indicate the voltages at which oxidation (lower lines) and reduction (top lines) is occurring. (A) A representative baseline current trace and color plot for the stimulated release of dopamine. The black dashed line $(t=0)$ indicates time of stimulation. (B) Representation of iontophoretic ejection of $3 \mu \mathrm{M}$ AP. The black dashed line $(t=0)$ indicates the application of a positive current to the barrel. (C) Current trace and color plot for stimulated release after ejection seen in panel B. The black dashed line $(t=0)$ indicates time of stimulation. There is no change in the extracellular concentrations of dopamine seen in panels A and C elicited with a stimulation indicated by the black dashed line and $t=0$ for each trace.

take advantage of the finely controlled drug delivery enabled by iontophoresis, we are interested in monitoring the biological effects of a single 30 s ejection and thus may not always reach this steady state at the site of interest, as will be evident later.

For a substance monitored by cyclic voltammetry with the carbon fiber, the amplitude of the voltammetric current is expected to increase as the ejected substance diffuses from the ejection point down the length of the fiber and to remain constant once the diffusion distance exceeds the electrode length. The concentration measured is actually the average concentration along the length of the electrode determined by a gradient started at the iontophoretic tip, where the concentration is close to that in the barrel $(10 \mathrm{mM})$. For example, an electrode with a length of $30 \mu \mathrm{m}$ (as in Figure 1) reports a current that is proportional to a uniform concentration of $3 \mu \mathrm{M}$ across the surface of the electrode once steady state is reached. When the iontophoretic current is turned off, the analyte quickly diffuses away from the electrode. Within $120 \mathrm{~s}$, the iontophoresed substance can still be detected voltammetrically, but it has diluted to a concentration that is $\sim 2 \%$ of its steady-state value during ejection.

\section{Effects of EOF Marker on Stimulated Dopamine Release}

A common way to probe presynaptic factors that regulate neurotransmitter release is to examine the effects of added pharmacological agents on electrically evoked release (33-35). For example, the role of autoreceptors can be probed by examining stimulations before and after addition of receptor antagonists. However, before the iontophoretic method was used with electrical stimulation to probe presynaptic events at dopaminergic terminals, we had to ensure that delivery of the neutral marker substances did not affect dopamine release. In these experiments, AP or NPE was delivered for $30 \mathrm{~s}$, followed by a wait-period of $120 \mathrm{~s}$ before electrical stimulation of the medial forebrain bundle (MFB). Figure 2 shows the results of a typical experiment in a urethane anesthetized rat. A carbonfiber/iontophoresis probe was lowered into the striatum and a stimulating electrode was lowered into the MFB. Stimulated release $(60 \mathrm{~Hz}, 40$ pulses $)$ was evoked every 120 s until 10 consecutive maximal stimulations showed a similar maximal concentration (typically requiring 15 stimulations). A representative baseline trace and color plot are shown in Figure 2A. To test the effects of the EOF marker on dopamine release, AP was iontophoretically applied for $30 \mathrm{~s}$ at a location that exhibited reproducible stimulated release. The amount of AP delivered was monitored electrochemically, and the concentration versus time trace and color plot are shown in Figure 2B. Stimulation was repeated $120 \mathrm{~s}$ after AP delivery, and the maximum amplitude of released dopamine and its time course remained the 
(A)

Pre-saline

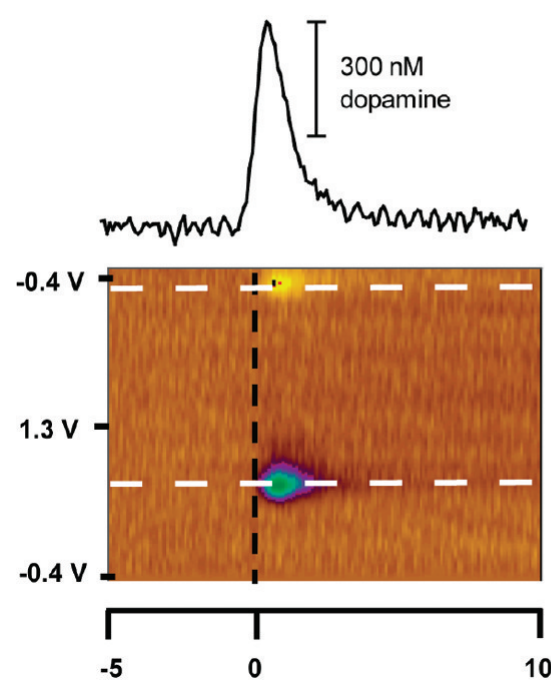

(B)
Iontophoresis of saline

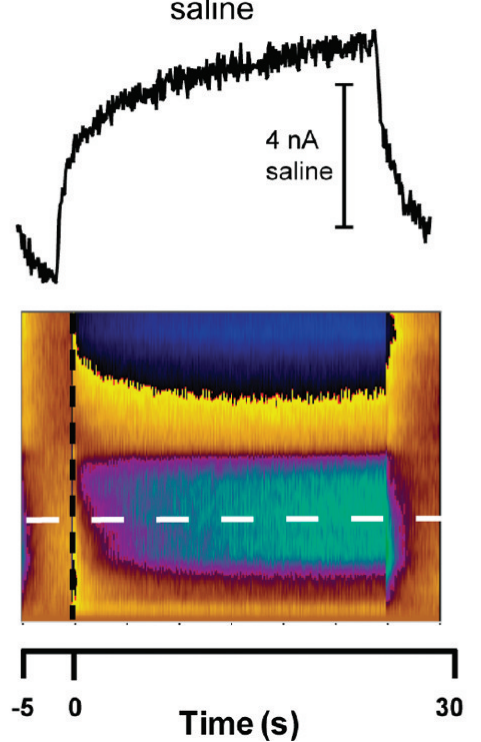

(C) Post-saline
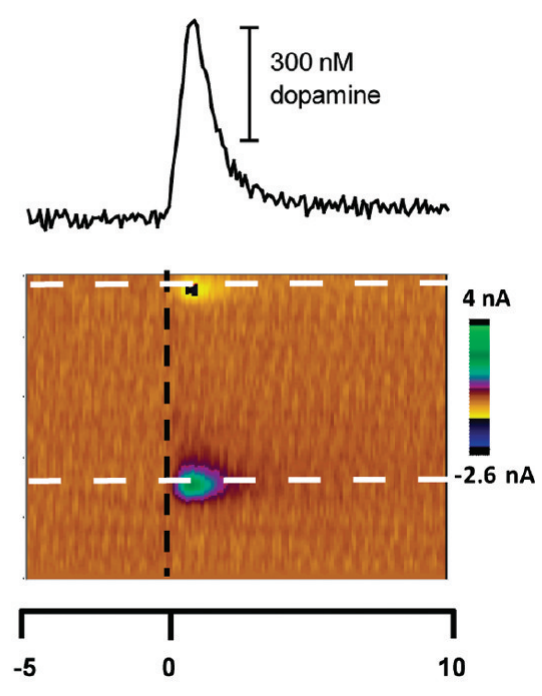

Figure 3. Effect of saline on stimulated dopamine release. The top panels show current as a function of time, while the lower panels are twodimensional color plots where current is shown in false color on the potential vs time axes. The white dashed lines on the color plots in panels A and $\mathrm{C}$ indicate the voltages at which oxidation (lower lines) and reduction (top lines) is occurring, whereas in panel $\mathrm{B}$, the dashed white line indicates the potential change observed due to the ejection of $\mathrm{NaCl}$. (A) A representative baseline current trace and color plot for the stimulated release of dopamine. The black dashed line $(t=0)$ indicates time of stimulation. (B) Representation of iontophoretic ejection of saline with high applied current. The black dashed line $(t=0)$ indicates the application of a positive current to the barrel. (C) Current trace and color plot for stimulated release after ejection seen in panel $\mathrm{B}$. The black dashed line $(t=0)$ indicates time of stimulation. There is no change in the extracellular concentrations of dopamine seen in panels A and C elicited with a stimulation indicated by the black dashed line and $t=0$ for each trace.

same. From the concentration of AP during its iontophoresis, $3 \mu \mathrm{M}$, we can estimate the concentration at the time of the stimulation to be $60 \mathrm{nM}(2 \%$ of the steadystate level). Stimulations were continued at 2 min intervals for $30 \mathrm{~min}$, and there was no significant change in the amount of dopamine released or rate of reuptake (Figure $2 \mathrm{C}, n=9, p>0.01$ ). The same experiment was performed for NPE, with no measurable difference observed from control $(n=4, p>0.01)$.

\section{Current Artifacts on Stimulated Dopamine Re- lease}

It is often noted in iontophoretic literature that current artifacts can be seen neurophysiologically due to the introduction of $\mathrm{Na}^{+}$and $\mathrm{Cl}^{-}$that are in the drug solution $(13,23)$. Although in our experiments we are not monitoring cell firing and are instead monitoring presynaptic release of dopamine, we wanted to ensure that current artifacts were not affecting our measured results. For these experiments, just as in the previous set of experiments, we adjusted the position of the carbon fiber/iontophoresis assembly so that it was in a location in the striatum that showed robust dopamine release. After establishing reproducible stimulated release of dopamine, we iontophoretically ejected $\mathrm{NaCl}(5 \mathrm{mM}$ in the barrel, made up in deionized water) for $30 \mathrm{~s}$. Although $\mathrm{NaCl}$ is not electroactive, by applying a large constant current ( $80 \mathrm{nA}$ ) we are able to detect a change in our background signal ( $\sim 4 \mathrm{nA}$ in this example) that evolves with time (Figure 3 ). The current is an indirect effect of the iontophoresis and its time course indicates that it reflects a change in the electrical double layer formed at the carbon-fiber electrode. While we cannot quantify the amount of $\mathrm{NaCl}$ delivered from this signal, it does serve to confirm that ejection occurred. Stimulated dopamine release was then measured $120 \mathrm{~s}$ after the $\mathrm{NaCl}$ ejection was terminated to ensure that it did not alter presynaptic release dynamics. The results from this experiment indicate that stimulated dopamine release is unaffected by large ejections of $\mathrm{NaCl}$ and are shown in Figure $3(n=4, p>0.05)$. The $\mathrm{NaCl}$ ejected with large currents does alter the double layer of the carbon fiber, although this is not seen with the lower ejection currents typically used.

\section{Marking of Electrode Placement by Iontophoresis of a Dye}

When dealing with small brain structures, it is crucial to know the location of the electrode. A common way to verify electrode placement is to remove the brain after the experiment is over for histology. For experiments using carbon-fiber microelectrodes, one approach is to electrolytically lesion the electrode. This has the shortcoming that the carbon fiber is destroyed during this 


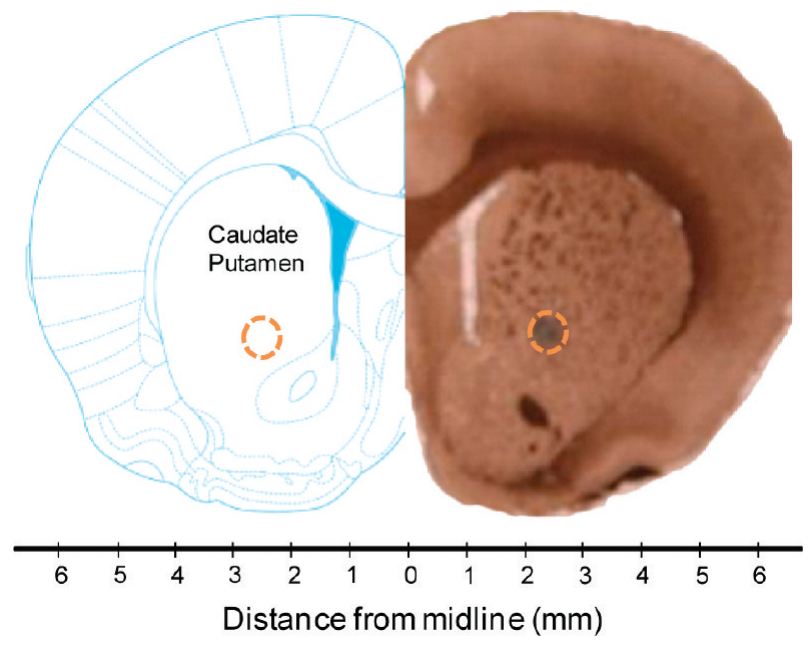

Figure 4. Confirmation of electrode placement into the striatum using iontophoresis of pontamine sky blue dye. After a $20 \mathrm{~min}$ ejection, the spot is $600 \mu \mathrm{m}$ in diameter. Left side of the figure shows region of interest labeled and circled with a dashed line. On the right, the spot from the ejection of dye can be seen in the circled region of interest. Adapted with permission from ref 45. Copyright 2007 Elsevier.

process and cannot be calibrated after the in vivo experiment. An alternate approach is to remove the electrode after the experiment for calibration and insert a tungsten wire to electrically mark the location of the previous electrode. Iontophoresis barrels provide a more convenient and precise method to mark electrode placement. Figure 4 shows the marking of electrode placement by delivering pontamine sky blue dye iontophoretically for $20 \mathrm{~min}$ at $40 \mathrm{nA}$ once the experiment was over. The length of ejection was chosen to ensure that a large enough spot was produced, given that iontophoresis is such a localized drug delivery mechanism. By using of one of the empty barrels to deliver a dye, the electrode placement can be accurately determined, while keeping the carbon fiber intact for postcalibration. Many other dyes can be used, such as alcian blue, methyl blue, fast green, and lucifer yellow, making the procedure compatible with any other immunohistochemistry that may be done postexperiment (23).

\section{Quantitative Iontophoresis of Nonelectroactive Drugs}

In our previous work, relative iontophoretic mobilities at capillary tips were obtained for electroactive molecules by measuring the ejected amounts at the adjacent carbon-fiber microelectrode (24). We found that neutral molecules such as AP can be ejected, establishing a role for EOF. We also established the role of EOF by demonstrating that iontophoretic and electrophoretic mobilities measured via capillary electrophoresis are correlated. Thus, at the tip of an iontophoresis pipet, delivery is controlled by both the migration of ions in an electric field and EOF. To obtain the iontophoretic mobility of electroinactive molecules such as the
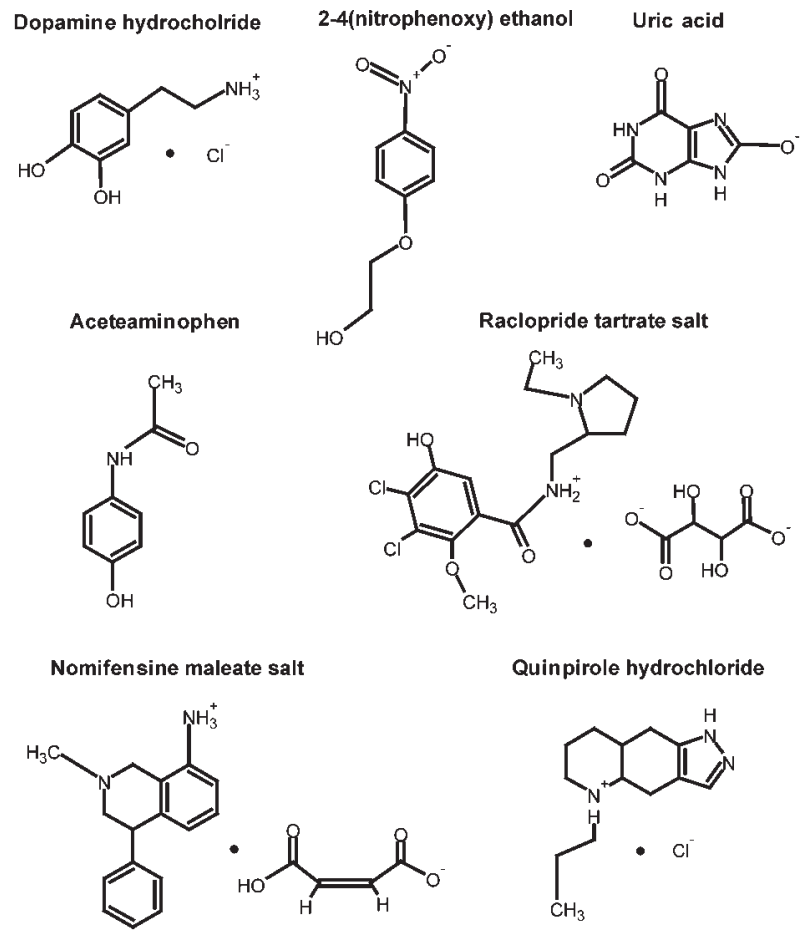

Figure 5. Chemical structures of compounds used for study. Shown are compounds with their associated anions. At $\mathrm{pH}$ 5.8, raclopride, nomifensine, quinpirole, and dopamine are all protonated based upon their $\mathrm{p} K_{\mathrm{a}}$ 's obtained on Scifinder. All solutions were made up as $10 \mathrm{mM}$ in $5 \mathrm{mM} \mathrm{NaCl}$ to ensure adequate electroosmotic flow and for buffering of the ionic strength.

dopaminergic drugs raclopride, quinpirole, and nomifensine (structures shown in Figure 5), the electrophoretic mobility through a capillary column was measured with UV detection. Retention times were used to compute the electrophoretic mobilities for dopamine, raclopride, quinpirole, nomifensine (all monocations), AP (a neutral molecule), and uric acid (a monoanion). The electrophoretic mobilities were then used to calculate the relative iontophoretic mobilities as shown in Figure 6. Thus, raclopride is ejected at a rate 1.68 times as fast as $\mathrm{AP}$, whereas quinpirole and nomifensine are ejected at a rate 2.18 and 2.24 times as fast as AP, respectively. With the knowledge of these ratios, the amount of an electroinactive molecule that is ejected can be calculated by the measured coejection of a neutral electroactive molecule from the same barrel. Note that the relative mobilities are in agreement with the expected charge (calculated from the relevant $\mathrm{p} K_{\mathrm{a}}$ 's) and the size of the molecules.

With an average ejection and an electrode with a length of $30 \mu \mathrm{m}$, the average concentration of AP across the carbon fiber is $3 \mu \mathrm{M}$ for the example shown in Figure 2. However, the concentration at the portion of the carbon fiber closest to the iontophoresis tip is very near that placed inside the barrel $(10 \mathrm{mM})$. Such high concentrations of drug could alter the sensitivity of the 


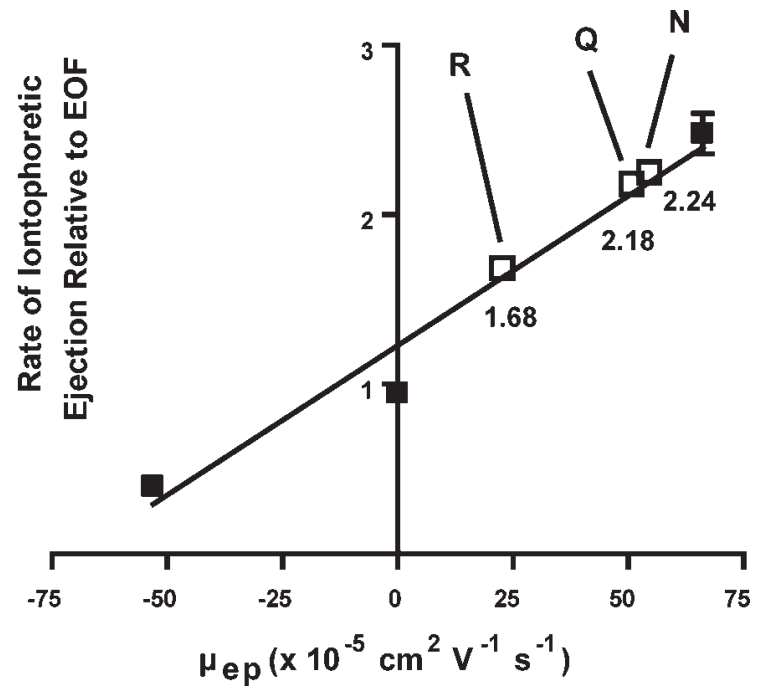

Figure 6. The electrophoretic mobilities of the electroactive compounds uric acid, AP, and DA ( $\square$ ) are positively correlated to previously reported iontophoretic rates. The linear regression from this correlation was used to determined iontophoretic rates relative to AP for raclopride (R), quinpirole (Q), and nomifensine $(\mathrm{N})(\square)$ based on their electrophoretic mobilities calculated by capillary electrophoresis.

electrode to dopamine. To evaluate this, we first calibrated a series of carbon-fiber iontophoresis probes to determine their sensitivity to dopamine. We then loaded one of the iontophoresis barrels with the EOF marker (AP) or AP and a drug of interest. While monitoring the response of AP with the carbon-fiber microelectrode, we continuously ejected the mixture into buffer for $40 \mathrm{~min}$ with a pump current sufficient to deliver approximately $10 \mu \mathrm{M}$ of AP. After the 40 min ejection and monitoring period, we calibrated the carbon-fiber electrodes' sensitivity to dopamine again and determined the ratio of the electrodes' post-iontophoresis sensitivity to pre-iontophoresis sensitivity. The results from these experiments are presented in Table 1. AP and NPE, which are both neutral and can be used as EOF markers, did not significantly alter the electrodes' response to dopamine. Pharmacological agents, such as nomifensine, quinpirole, and raclopride, have slight effects on the electrodes' response to dopamine. Note, however, that the ejection times used during these iontophoresis experiments (40 min per ejection) were considerably longer than would be used in most in vivo experiments (normally $30 \mathrm{~s}$ per ejection). Given the small effect observed, even with these prolonged iontophoresis conditions, the results demonstrate that iontophoresis of these drugs during in vivo experiments will not affect our dopamine measurements.

\section{Modulation of Neurotransmitter Release Using Quantitative Iontophoresis}

Dopamine release from terminals in the striatum is regulated by D2-autoreceptors (34). Quinpirole, a D2agonist, has been shown to decrease stimulated dopamine
Table 1. Effect of Iontophoresis on the Sensitivity of Carbon-Fiber Microelectrodes for Dopamine Detection

\begin{tabular}{lc}
\hline $\begin{array}{l}\text { solution ejected by } \\
\text { iontophoresis }\end{array}$ & $\begin{array}{c}\text { post-ionto/pre-ionto sensitivity } \\
(\mathrm{nA} / \mathrm{nM})\end{array}$ \\
\hline $\mathrm{AP}$ & $1.00 \pm 0.10$ \\
$\mathrm{AP}+\mathrm{NPE}$ & $1.01 \pm 0.07$ \\
$\mathrm{AP}+$ nomifensine & $0.86 \pm 0.08$ \\
$\mathrm{AP}+$ quinpirole & $0.91 \pm 0.08$ \\
$\mathrm{AP}+$ raclopride & $0.87 \pm 0.07$ \\
\hline
\end{tabular}

release in slices (36), and we wanted to show in vivo modulation with iontophoretic application of quinpirole. For these experiments, we adjusted the position of the carbon fiber/iontophoresis assembly so that it was in a location in the striatum that showed robust dopamine release (37). After establishing reproducible stimulated release of dopamine, we iontophoretically ejected quinpirole and AP from the same barrel for $30 \mathrm{~s}$. The local AP concentration was monitored by fast-scan cyclic voltammetry. The stimulation was repeated $120 \mathrm{~s}$ after the iontophoretic delivery terminated when AP had diminished to $\sim 2 \%$ of its concentration during iontophoretic application. From the AP concentration we can calculate that the local quinpirole concentration at the time of the stimulation was $88 \mathrm{nM}$. This value is near the $\mathrm{EC}_{50}$ $(60 \mathrm{nM})$ for quinpirole measured in brain slices (38).

Consistent with autoreceptor regulation, dopamine release was diminished (representative example in Figure 7). This experiment was repeated in six different rats with a different iontophoretic assembly in each animal. In these experiments, the amount of current used for ejection was adjusted so that the same amount of AP (and thus quinpirole) was ejected in each animal. The release amplitude was $63 \% \pm 5 \%(n=6)$ of its predrug value. The small error associated with these measurements highlights the advantage of using an electroactive marker, since it allows for adjustment to the applied iontophoretic current so that uniform amounts of quinpirole are ejected. Thus, compensation can be made for the variability inherent to each iontophoretic barrel. Because autoreceptors and reuptake processes appear to be linked, we also examined the clearance rates of dopamine after stimulation. While a trend toward faster reuptake rates was observed after quinpirole ejections, there was not a statistically significant decrease in $t_{1 / 2}$.

Stimulations were repeated at 2 min intervals, and the amplitude returned to its original value within three to five stimulations after the initial iontophoretic application. In addition, when the electrode was lowered $400 \mu \mathrm{m}$, release similar to that seen in the absence of drug was observed. Since the amounts introduced by iontophoresis are microscopic, it would not be expected to exert an effect over a region much larger than that immediately around the electrode. It is worth noting 
(A)

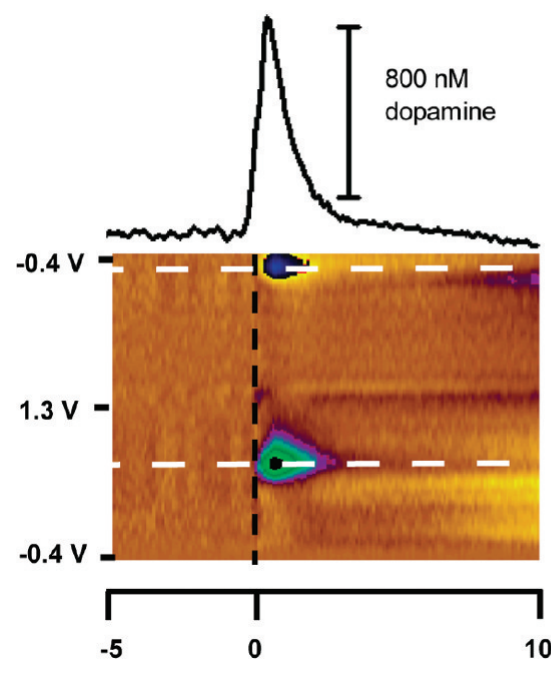

(B)
lontophoresis of quinpirole

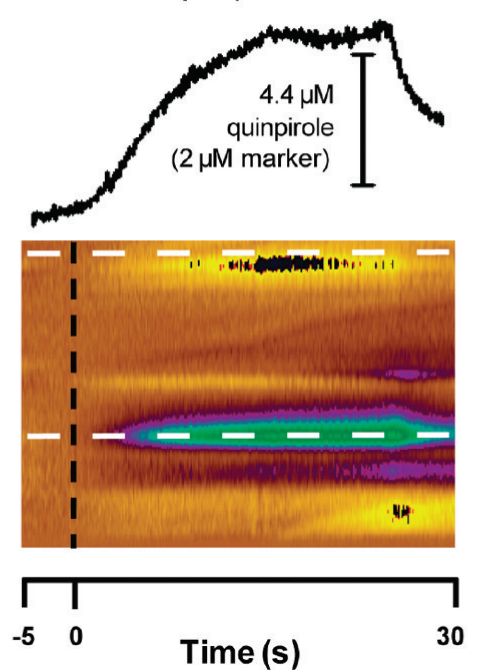

(C) Post-drug

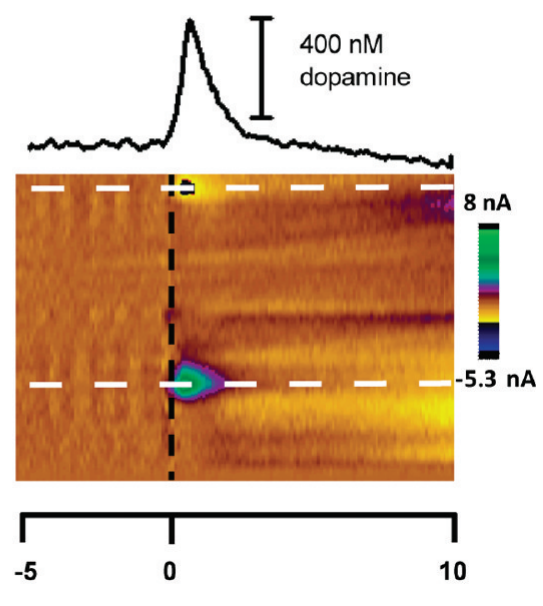

Figure 7. Stimulated dopamine release in an anesthetized animal before and after a localized ejection of solution containing both AP and quinpirole. The top panels show current as a function of time, while the lower panels are two-dimensional color plots where current is shown in false color on the potential vs time axes. The white dashed lines on the color plots indicate the voltages at which oxidation (lower lines) and reduction (top lines) is occurring. (A) A representative baseline current trace and color plot for the stimulated release of dopamine. The black dashed line indicates the time of stimulation. (B) Representation of iontophoretic ejection of AP and quinpirole. The black dashed line $(t=0)$ indicates the application of a positive current to the barrel. The measured signal is due solely to AP and is used to estimate the concentration of quinpirole. Here, $2 \mu \mathrm{M}$ AP is the average concentration across the electrode, and is equivalent to $4.4 \mu \mathrm{M}$ quinpirole. (C) Current trace and color plot for stimulated release $120 \mathrm{~s}$ after ejection seen in panel B. At the time of stimulation (black dashed line), the concentration of AP has decreased to $2 \%$ of its original value, corresponding to a decrease in quinpirole concentration to $88 \mathrm{nM}$. The extracellular concentration of dopamine seen in panel $\mathrm{C}$ is less than half the concentration initially seen in panel $\mathrm{A}$. In both panels $\mathrm{A}$ and $\mathrm{C}$ the time of stimulation is indicated by the black dashed line and $t=0$ for each trace.

that most iontophoresis experiments done previously used ejections much longer than $30 \mathrm{~s}$, some delivering drug for tens of minutes (39). Such ejections seem unnecessary in light of the results presented here. Indeed, the ability to make multiple ejections at the same or different sites enables multiple concentrations of drugs to be examined in a single animal. Systemic doses do not allow this type of flexibility, clearly highlighting one of the major advantages of iontophoresis.

\section{Modulation of Other Dopaminergic Presynaptic Processes}

Similar experiments were done with raclopride, a D2receptor antagonist, which can block dopamine autoreceptor function, leading to an increase in release, as well as nomifensine, a dopamine reuptake inhibitor that increases the amount of time required for dopamine to clear the synapse. The results from these experiments showed that raclopride increased stimulated dopamine release to $270 \% \pm 40 \%(n=5)$ of its predrug value. Raclopride also caused a decrease in reuptake rate, as indicated by the increased $t_{1 / 2}$, from $0.63 \pm 0.03 \mathrm{~s}$ to $0.96 \pm 0.05 \mathrm{~s}$. This result is consistent with previous work where systemic injections of a D2-antagonist affected both release and reuptake $(34,40)$ and further supports the idea that autoreceptor antagonists increase evoked DA levels by decreasing reuptake in a complex signaling process. Similarly, nomifensine delivery resulted in a $187 \% \pm 13 \%$ increase in stimulated dopamine release and an increased $t_{1 / 2}$ from $0.63 \pm 0.03 \mathrm{~s}$ to $1.36 \pm 0.05 \mathrm{~s}$ (Figure 8). The modulations observed due to localized application of all three drugs is consistent with those found for systemic injections (41).

\section{Rapid Modulation of Dopamine Release and Reuptake}

One of the advantages of localized drug delivery is that drug effects are observed quickly. Figure 9 demonstrates rapid modulation of dopamine release and reuptake by using a D2 agonist, D2 antagonist, and dopamine reuptake blocker. Quinpirole, a D2 agonist, was delivered to attenuate dopamine release. As can be seen from Figure 9A, a decrease in release is observed immediately after the $30 \mathrm{~s}$ ejection. In this representative experiment, the signal slowly returned to baseline over 10 min. Raclopride, a D2 antagonist, which blocks autoreceptors on dopamine terminals, quickly increased the amount of dopamine release observed by 3 -fold. In contrast to quinpirole, this effect remained steady for over $10 \mathrm{~min}$. To observe effects on dopamine reuptake, we used the dopamine reuptake inhibitor nomifensine. As expected from previous findings, the reuptake blocker increased the amount of dopamine release and slowed reuptake. This can be seen in the color plots 


\section{(A) Pre-drug}

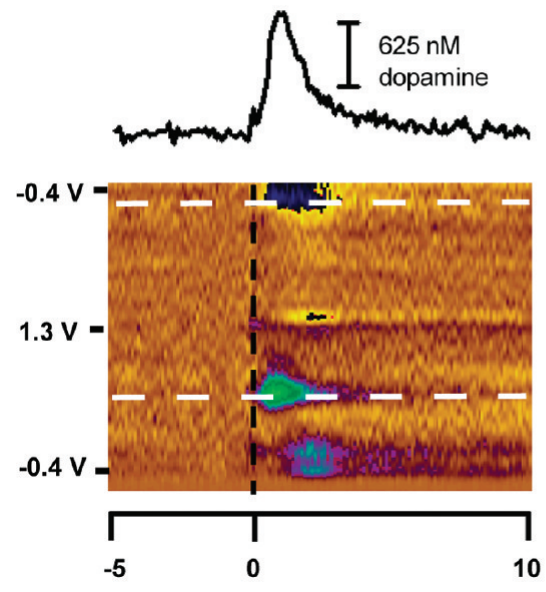

(B)

lontophoresis of nomifensine

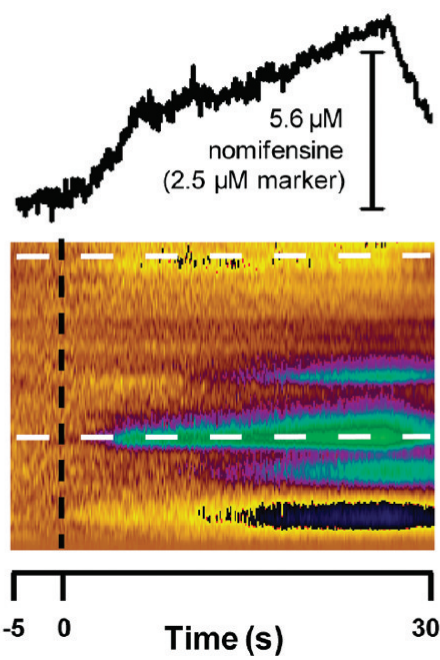

(C) Post-drug

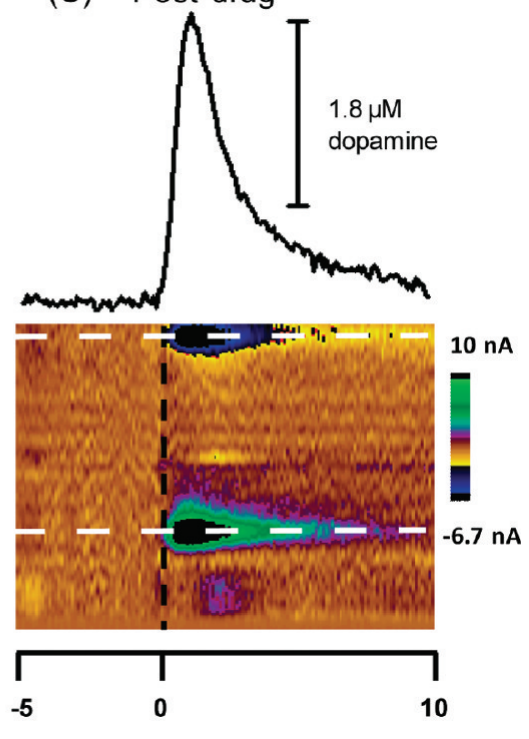

Figure 8. Stimulated dopamine release in an anesthetized animal before and after a localized ejection of solution containing both AP and nomifensine. The top panels show current as a function of time, while the lower panels are two-dimensional color plots where current is shown in false color on the potential vs time axes. The white dashed lines on the color plots indicate the voltages at which oxidation (lower lines) and reduction (top lines) is occurring. (A) A representative baseline current trace and color plot for the stimulated release of dopamine. The black dashed line is the time to stimulation. (B) Representation of iontophoretic ejection of AP and nomifensine. The black dashed line $(t=0)$ indicates the application of a positive current to the barrel. Note that the measured signal is due solely to AP and is used to estimate the concentration of nomifensine. Here, $2.5 \mu \mathrm{M}$ AP is the average concentration across the electrode and is equivalent to $5.6 \mu \mathrm{M}$ nomifensine. (C) Current trace and color plot for stimulated release $120 \mathrm{~s}$ after ejection seen in panel B. At the time of stimulation (black dashed line), the concentration of AP has decreased to $2 \%$ of its original value, corresponding to a decrease in nomifensine concentration to $112 \mathrm{nM}$. The extracellular concentration of dopamine seen in panel $\mathrm{C}$ is significantly increased and the clearance time is also increased, indicating a change in reuptake kinetics. In both panels $\mathrm{A}$ and $\mathrm{C}$, the time of stimulation is indicated by the black dashed line and $t=0$ for each trace.

(A)

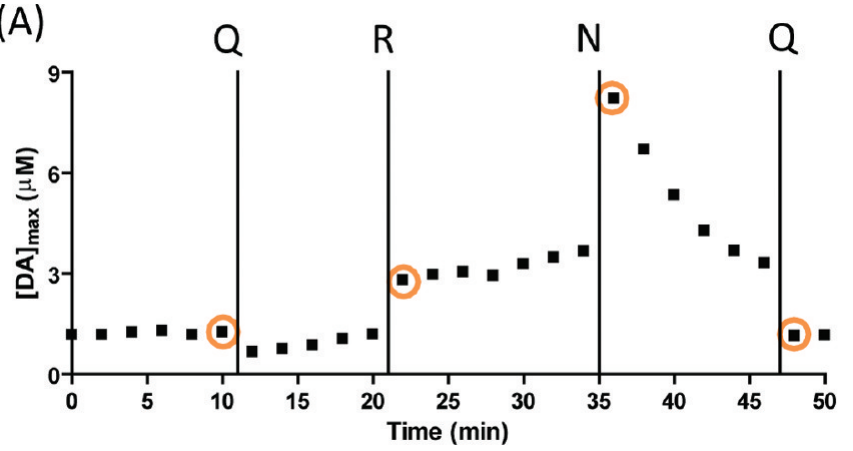

(B)
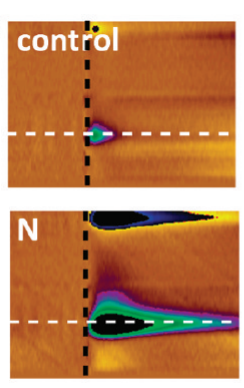
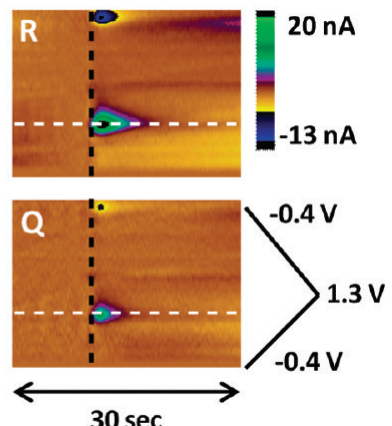

Figure 9. Rapid modulation of DA autoreceptors using quinpirole and raclopride and dopamine transporter using nomifensine. (A) Stimulated release of DA is recorded every $120 \mathrm{~s}$, and plotted is the maximum amount of dopamine overflow recorded from each stimulation. The circles denote the time points at which the color plots in panel B were taken. With the application of quinpirole (Q), raclopride (R), and nomifensine $(\mathrm{N})$ at the time represented with the vertical dashed line, there was a change in DA signal seen at the next stimulation. (B) Color plots for the stimulated release of dopamine before application of any drugs and after the administration of each drug. The duration of the dopamine signal (white dashed line) after stimulation (black dashed line) is indicative of the reuptake kinetics.

shown at each point of modulation in Figure 9B. The dopamine signal apparent in the color plots has a longer duration once nomifensine is on board. This effect, however, is shorter lived than the raclopride effect, and over the course of $10 \mathrm{~min}$, the signal returns to what it was before nomifensine ejection. To see whether the raclopride and nomifensine effects could be reversed, quinpirole was reapplied, resulting in dopamine release returning back to the original baseline. In addition to demonstrating that iontophoresis can be used to quickly and robustly modulate dopamine release and reuptake, it also gives insight into the different rates of unbinding for each of these drugs. The results show that nomifensine has a shorter-lasting effect than quinpirole and raclopride, consistent with studies that show nomifensine has the fastest off rate from its binding site to striatal membranes (when adjusted for temperature) $(42-44)$. 


\section{Conclusions}

The results presented characterize and validate the use of an electroactive EOF marker for quantitative iontophoresis using carbon-fiber microelectrode assemblies. Nanomolar concentrations of raclopride, quinpirole, and nomifensine can be delivered by monitoring the coejection of the EOF marker. The effects of local delivery of these drugs can then be monitored by measuring electrically evoked dopamine release before and after drug. We show that a short $30 \mathrm{~s}$ ejection is sufficient to affect autoreceptor regulation and reuptake of dopamine. Additionally, electrode placement can be verified by iontophoresis of a dye, such as pontamine sky blue. These experiments highlight the advantages of iontophoresis: quick, local, and selective receptor modulation. The use of carbon-fiber microelectrodes and an EOF marker enables real-time measurements of drug delivery, eliminating confounds from faulty ejections and differences in doses. These modifications improve the technique of iontophoresis for in vivo neuropharmacological experiments.

\section{Methods}

\section{Chemicals}

Unless noted, all chemicals were purchased from SigmaAldrich (St. Louis, MO) and used as received. Chemical structures of the species used are shown in Figure 5. Solutions were prepared using deionized water. A physiological buffer solution (15 mM TRIS, $126 \mathrm{mM} \mathrm{NaCl}, 2.5 \mathrm{mM} \mathrm{KCl}, 25 \mathrm{mM}$ $\mathrm{NaHCO}_{3}, 2.4 \mathrm{mM} \mathrm{CaCl}_{2}, 1.2 \mathrm{mM} \mathrm{NaH}_{2} \mathrm{PO} 4,1.2 \mathrm{mM} \mathrm{MgCl}_{2}$, $2.0 \mathrm{mM} \mathrm{Na} \mathrm{SO}_{4}$ ), $\mathrm{pH} 7.4$, was used in all calibration experiments.

\section{Animals and Surgery}

Male Sprague-Dawley rats (225-350 g; Charles River, Wilmington, MA) were anesthetized with urethane $(1.5 \mathrm{~g} / \mathrm{kg}$, i.p.) and placed in a stereotaxic frame (Kopf, Tujunga, CA). Holes were drilled in the skull on the right hemisphere for the working and stimulating electrodes at coordinates selected from the atlas of Paxinos and Watson (45). $\mathrm{A} \mathrm{Ag} / \mathrm{AgCl}$ reference electrode was inserted in the left hemisphere. The carbon-fiber iontophoresis probe was placed in the striatum (AP $+1.2 \mathrm{~mm}, \mathrm{ML}+2.0 \mathrm{~mm}$, and $\mathrm{DV}-4.5$ to $-6 \mathrm{~mm}$ ). The stimulating electrode was placed in the medial forebrain bundle (AP $-2.8 \mathrm{~mm}, \mathrm{ML}+1.7 \mathrm{~mm}$, and $\mathrm{DV}-8.5 \mathrm{~mm}$ ). The carbon-fiber and stimulating electrodes were individually adjusted in the dorsal-ventral coordinate to locate the optimal locations for stimulated dopamine release.

\section{Electrical Stimulation}

An untwisted bipolar stimulating electrode (Plastics One, Roanoke, VA) was used to stimulate dopaminergic neurons using a pair of linear constant current stimulus isolators (model NL80A, NeuroLog System, Digitimer Ltd., U.K.). The stimulation train consisted of 40 biphasic pulses $( \pm 300$ $\mu \mathrm{A}, 2 \mathrm{~ms} /$ phase unless otherwise noted) applied at $60 \mathrm{~Hz}$. The pulses were generated by a computer and applied between the cyclic voltammograms to avoid electrical interference.

\section{Iontophoresis Probes}

A glass capillary (part no. $624503,0.60 \mathrm{~mm}$ o.d., $0.4 \mathrm{~mm}$ i.d., 4 in. long, A-M Systems, Sequim, WA) was loaded with a carbon fiber (T-650, Thornel, Amoco Corp., Greenville, SC) that served as the working electrode. This capillary containing the carbon fiber was then inserted into one barrel of a 4-barrel capillary (part no. 50644, $1 \mathrm{~mm}$ o.d., $0.75 \mathrm{~mm}$ i.d., four barrel GF pipettes, 4 in. long, Stoelting Co., Wood Dale IL). The four barrel assembly contained glass filaments (GF) in each barrel that aid in filling the barrel by capillary action. The capillaries were bundled together with heat shrink and tapered to a sharp tip using a micropipet puller (Narashige, Tokyo, Japan) with a two-step pull process. The protruding carbon fiber was cut to a length between 30 and $50 \mu \mathrm{m}$ by careful use of a scalpel under a $10 \times$ microscope objective. The resulting probe consists of a glass-encased carbon fiber that is $5-7 \mu \mathrm{m}$ in diameter and three iontophoretic barrels each about $1 \mu \mathrm{m}$ in diameter. Before use, the barrel containing the carbon fiber was backfilled with electrolyte ( $4 \mathrm{M}$ potassium acetate, $150 \mathrm{mM}$ potassium chloride) and fitted with wires for electrical contact. The remaining barrels for iontophoresis were filled with solutions containing reagents to be ejected.

\section{Electrochemical Data Acquisition and Presentation}

Cyclic voltammograms were acquired using data-acquisition hardware and local software written in LabVIEW (National Instruments, Austin, TX). The cyclic voltammetry waveform was generated and the voltammetric signal was acquired with a computer interface board, the PCI-6052E (National Instruments). A PCI-6711E D/A board (National Instruments) was used to synchronize waveform application and data acquisition and to trigger the iontophoretic current applied and the loop injector in the flow injection apparatus. The voltammetric waveform was input into a custom-built instrument for application to the electrochemical cell and current transduction (University of North Carolina at Chapel Hill, Department of Chemistry Electronics Facility). After data collection, background subtraction, signal averaging, and digital filtering (low-pass filtered at $2 \mathrm{kHz}$ ) were all done under software control.

For all experiments, a triangular waveform was applied with a scan rate of $400 \mathrm{~V} \mathrm{~s}^{-1}$ with a rest potential of $-0.4 \mathrm{~V}$ versus a $\mathrm{Ag} / \mathrm{AgCl}$ reference electrode between scans and a linear scan to $1.3 \mathrm{~V}$, followed by a scan back to the rest potential. The scans were repeated every $100 \mathrm{~ms}$, and collection was typically for 15-60 s. This large amount of data is presented as a color plot, with the applied voltage plotted on the ordinate, time on the abscissa, and measured current in false color.

\section{Iontophoresis Ejections}

Characterization studies involving the effects of the neutral marker molecule on stimulated dopamine release were done with solutions made up at $10 \mathrm{mM}$ concentrations of acetaminophen and 2-4(nitrophenoxy) ethanol in $5 \mathrm{mM} \mathrm{NaCl}$. The effects of saline were studied with $5 \mathrm{mM} \mathrm{NaCl}$ solutions. For studies involving the modulation of stimulated dopamine release, each barrel of the iontophoresis assembly was filled with either raclopride tartrate salt, nomifensine maleate salt, or quinpirole hydrochloride and the EOF marker, usually AP, at concentrations of $\sim 10 \mathrm{mM}$ each in $5 \mathrm{mM} \mathrm{NaCl}$ at pH 5.8 . 
These concentrations of drug and supporting electrolyte were chosen for the following reasons: Electrolyte is needed to facilitate conductivity and to ensure adequate and reproducible EOF without being so high that EOF is suppressed (46); therefore $5 \mathrm{mM} \mathrm{NaCl}$ was chosen as a compromise between the two limits. Traditionally, drug concentrations are higher than used here and barrels are sometimes loaded with drugs at concentrations of $250 \mathrm{mM}$. However, given that we are able to electrochemically monitor drug ejection, we determined that $10 \mathrm{mM}$ is sufficiently concentrated to get observable ejection and observe effects on stimulated dopamine release. Ejection currents were delivered by a constant current source designed for iontophoresis (Neurophore, Harvard Apparatus, Holliston, MA). For each barrel, an ejection current (between 5 and $40 \mathrm{nA}$ ) was selected by evaluating ejections ( 30 s duration) that gave a measurable voltammetric signal for the EOF marker (average peak current of 5-30 nA at the peak potential in the voltammogram). A current of $0 \mathrm{nA}$ was applied between ejections.

To minimize electrical cross-talk between the electrochemical and iontophoretic electrodes, both systems had a common ground. The reference electrode served as the return for the iontophoresis currents and was tied to ground. The potential of the working electrode was controlled by applying the voltage to the noninverting input of the current transducer.

\section{Calibrations}

The response of the carbon fiber electrode in the iontophoresis probe was calibrated in a flow injection analysis system after in vivo use (47). The probe was positioned at the outlet of a six-port rotary valve. A loop injector was mounted on an actuator (Rheodyne model 7010 valve and 5701 actuator) that was used with a $12-\mathrm{V}$ DC solenoid valve kit (Rheodyne, Rohnert Park, CA) to introduce the analyte to the surface of the electrode. The linear flow velocity $\left(1.0 \mathrm{~cm} \mathrm{~s}^{-1}\right)$ was controlled with a syringe infusion pump (Harvard Apparatus model 940, Holliston, MA). The voltammetric current was measured at the peak potential for each analyte that was evaluated at four concentrations.

\section{Capillary Electrophoresis Experiments}

A home-built CE system equipped with an absorbance detector and a $30 \mathrm{kV}$ power supply was employed. Absorbance traces were collected using a custom written LabVIEW program (courtesy of Professor James Jorgensen, UNC-CH). Separations were carried out in a $50 \mu \mathrm{m}$ diameter fused silica capillary, $96.0 \mathrm{~cm}$ in total length, with the UV detector placed $87.5 \mathrm{~cm}$ from the inlet. Experiments were done in cationic mode (the anode at the inlet and cathode at the outlet). Samples were run at a concentration of $2 \mathrm{mM}$ in $17 \mathrm{mM}$ PBS (phosphate-buffered saline, made up of $0.25 \%$ monosodium phosphate and $0.04 \%$ disodium phosphate) with a $\mathrm{pH}$ of 5.8 as in the iontophoresis experiments. UV detection was measured at 195 and $240 \mathrm{~nm}$ and electrophoretic mobilities were calculated as previously described (24).

\section{Histology}

After animal experiments were complete, pontamine sky blue was loaded into one of the empty iontophoresis barrels to mark electrode location. The dye was ejected by applying 40 $\mathrm{nA}$ of current for $20 \mathrm{~min}$. The animals were euthanized, and brains were removed from the skull and stored in $10 \%$ formaldehyde for at least 3 days. Brains were coronally sectioned into $40-50 \mu \mathrm{m}$ thick slices with a cryostat and visualized under a stereoscope equipped with a camera.

\section{Author Information}

\section{Corresponding Author}

*To whom correspondence should be addressed. E-mail address: rmw@unc.edu. Phone: 919-962-1472. Fax: 919962-2388.

\section{Author Contributions}

Natalie R. Herr performed experiments for Figures 1, 2, 4-9 and wrote the majority of the manuscript. Anna M. Belle performed experiments for Figure 3 and Table 1 and helped with writing of the manuscript. Kevin B. Daniel performed experiments for Table 1 and helped with writing of the manuscript. Regina M. Carelli and R. Mark Wightman are the PIs on the project and assisted with writing of the manuscript.

\section{Funding Sources}

We acknowledge financial support from NIH (NS15841 to R.M.W. and DA17318 to R.M.C. and R.M.W.).

\section{Acknowledgment}

The authors acknowledge Dr. Stephen Feldberg for guiding our thinking on the transport of material away from the electrode and Elyse Dankoski for the critical review of this manuscript.

\section{Abbreviations}

AP, acetaminophen; EOF, electroosmotic flow; NPE, 2-(4nitrophenoxy) ethanol; raclopride, raclopride tratrate salt; nomifensine, nomifensine maleate salt; quinpirole, quinpirole hydrochloride.

\section{References}

1. Hicks, T. P. (1984) The history and development of microiontophoresis in experimental neurobiology. Prog. Neurobiol. 22, 185-240.

2. Nastuk, W. L. (1953) Membrane potential changes at a single muscle endplate produced by transitory application of acetylcholine with an electrically controlled microjet. Fed. Proc. 102.

3. Del Castillo, J., and Katz, B. (1955) On the localization of acetylcholine receptors. J. Physiol. 128, 157-181.

4. Del Castillo, J., and Katz, B. (1956) Localization of active spots within the neuromuscular junction of the frog. J. Physiol. $132,630-649$.

5. Axelsson, J., and Thesleff, S. (1959) A study of supersensitivity in denervated mammalian skeletal muscle. J. Physiol. 147, 178-193.

6. Miledi, R. (1960) The acetylcholine sensitivity of frog muscle fibres after complete or partial devervation. J. Physiol. $151,1-23$. 
7. Curtis, D. R., and Eccles, R. M. (1958) The excitation of Renshaw cells by pharmacological agents applied electrophoretically. J. Physiol. 141, 435-445.

8. Curtis, D. R., Phillis, J. W., and Watkins, J. C. (1960) The chemical excitation of spinal neurones by certain acidic amino acids. J. Physiol. 150, 656-682.

9. Curtis, D. R., and Koizumi, K. (1961) Chemical transmitter substances in brain stem of cat. J. Neurophysiol. 24, 80-90.

10. Krnjevic, K., Laverty, R., and Sharman, D. F. (1963) Iontophoretic release of adrenaline, noradrenaline and 5-hydroxytryptamine from micropipettes. Br. J. Pharmacol. Chemother. 20, 491-496.

11. Krnjevic, K., Mitchell, J. F., and Szerb, J. C. (1963) Determination of iontophoretic release of acetylcholine from micropipettes. J. Physiol. 165, 421-436.

12. Crawford, J. M., and Curtis, D. R. (1964) The excitation and depression of mammalian cortical neurones by amino acids. Br. J. Pharmacol. Chemother. 23, 313-329.

13. Curtis, D. R., Nastuk, W. L. (1964) Micro-electrophoresis, in Physical Techniques in Biological Research, pp 144-190, Academic Press, New York.

14. Bradley, P. B., and Candy, J. M. (1970) Iontophoretic release of acetylcholine, noradrenaline, 5-hydroxytryptamine and D-lysergic acid diethylamide from micropipettes. Br. J. Pharmacol. 40, 194-201.

15. Bloom, F. E. (1974) To spritz or not to spritz: The doubtful value of aimless iontophoresis. Life Sci. 14, 1819-1834.

16. Simmonds, M. A. (1974) Quantitative evaluation of responses to microiontophoretically applied drugs. Neuropharmacology 13, 401-406.

17. Freedman, R., Hoffer, B. J., and Woodward, D. J. (1975) A quantitative microiontophoretic analysis of the responses of central neurones to noradrenaline: Interactions with cobalt, manganese, verapamil and dichloroisoprenaline. Br. J. Pharmacol. 54, 529-539.

18. Purves, R. D. (1977) The release of drugs from iontophoretic pipettes. J. Theor. Biol. 66, 789-798.

19. Purves, R. D. (1979) The physics of iontophoretic pipettes. J. Neurosci. Methods 1, 165-178.

20. Purves, R. (1980) Ionophoresis - Progress and pitfalls. Trends Neurosci. 3, 245-247.

21. Purves, R. D. (1980) Effect of Drug Concentration on Release from Ionophoretic Pipettes. J. Phys.-London 300, P72-P73.

22. Armstrong-James, M., Fox, K., Kruk, Z. L., and Millar, J. (1981) Quantitative ionophoresis of catecholamines using multibarrel carbon fibre microelectrodes. J. Neurosci. Methods 4, 385-406.

23. Stone, T. W. (1985) Microiontophoresis and Pressure Ejection, John Wiley \& Sons, London.

24. Herr, N. R., Kile, B. M., Carelli, R. M., and Wightman, R. M. (2008) Electroosmotic Flow and Its Contribution to Iontophoretic Delivery. Anal. Chem. 80, 8635-8641.

25. Kiyatkin, E. A., Kiyatkin, D. E., and Rebec, G. V. (2000) Phasic inhibition of dopamine uptake in nucleus accumbens induced by intravenous cocaine in freely behaving rats. Neuroscience 98, 729-741.
26. Kiyatkin, E. A., and Rebec, G. V. (1996) Dopaminergic modulation of glutamate-induced excitations of neurons in the neostriatum and nucleus accumbens of awake, unrestrained rats. J. Neurophys. 75, 142-153.

27. Kiyatkin, E. A., and Rebec, G. V. (1997) Iontophoresis of amphetamine in the neostriatum and nucleus accumbens of awake, unrestrained rats. Brain Res. 771, 14-24.

28. Kiyatkin, E. A., and Rebec, G. V. (1999) Striatal neuronal activity and responsiveness to dopamine and glutamate after selective blockade of D1 and d2 dopamine receptors in freely moving rats. J. Neurosci. 19, 3594-3609.

29. Kiyatkin, E. A., and Rebec, G. V. (1999) Modulation of striatal neuronal activity by glutamate and GABA: iontophoresis in awake, unrestrained rats. Brain Res. 822, 88-106.

30. Kiyatkin, E. A., and Rebec, G. V. (2000) Dopamineindependent action of cocaine on striatal and accumbal neurons. Eur. J. Neurosci. 12, 1789-1800.

31. Rebec, G. V. (1998) Real-time assessments of dopamine function during behavior: single-unit recording, iontophoresis, and fast-scan cyclic voltammetry in awake, unrestrained rats. Alcohol.: Clin. Exp. Res. 22, 32-40.

32. Rice, M. E., Nicholson, C. (1995) Diffusion and ion shifts in the brain extracellular microenvironment and their relevance for voltammetric measurements, in Voltammetric methods in brain Systems, p 27, Humana Press, Totowa, NJ.

33. Limberger, N., Trout, S. J., Kruk, Z. L., and Starke, K. (1991) "Real time" measurement of endogenous dopamine release during short trains of pulses in slices of rat neostriatum and nucleus accumbens: Role of autoinhibition. Naunyn-Schmiedebergs Arch. Pharmacol. 344, 623-629.

34. Benoit-Marand, M., Borrelli, E., and Gonon, F. (2001) Inhibition of dopamine release via presynaptic $\mathrm{D} 2$ receptors: Time course and functional characteristics in vivo. J. Neurosci. 21, 9134-9141.

35. Phillips, P. E., Hancock, P. J., and Stamford, J. A. (2002) Time window of autoreceptor-mediated inhibition of limbic and striatal dopamine release. Synapse 44, 15-22.

36. Kennedy, R. T., Jones, S. R., and Wightman, R. M. (1992) Dynamic observation of dopamine autoreceptor effects in rat striatal slices. $J$. Neurochem. 59, 449-455.

37. Moquin, K. F., and Michael, A. C. (2009) Tonic autoinhibition contributes to the heterogeneity of evoked dopamine release in the rat striatum. J. Neurochem. 110, 14911501 .

38. Joseph, J. D., Wang, Y. M., Miles, P. R., Budygin, E. A., Picetti, R., Gainetdinov, R. R., Caron, M. G., and Wightman, R. M. (2002) Dopamine autoreceptor regulation of release and uptake in mouse brain slices in the absence of D(3) receptors. Neuroscience 112, 39-49.

39. Cheer, J. F., Aragona, B. J., Heien, M. L., Seipel, A. T., Carelli, R. M., and Wightman, R. M. (2007) Coordinated accumbal dopamine release and neural activity drive goaldirected behavior. Neuron 54, 237-244.

40. Wu, Q., Reith, M. E. A., Walker, Q. D., Kuhn, C. M., Carroll, F. I., and Garris, P. A. (2002) Concurrent autoreceptor-mediated control of dopamine release and uptake during neurotransmission: An in vivo voltammetric study. J. Neurosci. 22, 6272-6281. 
ACS Chemical

NeuroScience pubs.acs.org/acschemicalneuroscience

Article

41. Kita, J. M., Parker, L. E., Phillips, P. E., Garris, P. A., and Wightman, R. M. (2007) Paradoxical modulation of short-term facilitation of dopamine release by dopamine autoreceptors. J. Neurochem. 102, 1115-1124.

42. Levant, B., Grigoriadis, D. E., and DeSouza, E. B. (1992) Characterization of [3H]quinpirole binding to D2-like dopamine receptors in rat brain. J. Pharm. Exp. Then. 262, 929-935.

43. Dewar, K. M., Montreuil, B., Grondin, L., and Reader, T. A. (1989) Dopamine D2 receptors labeled with $[3 \mathrm{H}]$ raclopride in rat and rabbit brains. Equilibrium binding, kinetics, distribution and selectivity. J. Pharm. Exp. Then. 250, 696-706.

44. Dubocovich, M. L., and Zahniser, N. R. (1985) Binding characteristics of the dopamine uptake inhibitor $[3 \mathrm{H}]$ nomifensine to striatal membranes. Biochem. Dharmacol. 34, 1137-1144.

45. Paxinos, G., Watson, C. (2007) The Rat Brain in Stereoataxic Coordinates, Elsevier Academic Press, Boston, MA.

46. Gazes, J. (2001) Encyclopedia of Chromatography, Mareel Dekker, New York.

47. Kristensen, E. W., Wilson, R. L., and Wightman, R. M. (1986) Dispersion in flow injection analysis measured with microvoltammetric electrodes. Anal. Chem. 58, 986-988.

Note Added after ASAP Publication

This article was published on July 28, 2010 before final corrections were completed. The corrected version was published on July 30, 2010.

2010 American Chemical Society

638

DOI: 10.1021/cn100056r | ACS Chem. Neurosci. (2010), 1, 627-638 